\title{
Root Canal Treatment of a Mandibular Second Molar with a Reverse Anatomy
}

\author{
Ramesh Halebathi Giriyappa, Kishore Kumar Majety, Bhavana Gandhi
}

\begin{abstract}
The complex anatomy of the root canal system takes up many configurations and variations are not uncommon. A clinician should be aware of the normal, so as to recognize these aberrations in the anatomy. Successful root canal therapy is achieved by thorough shaping and cleaning of the root canal system followed by obturation of the radicular space. The aim of the present article is to describe a case of a mandibular second molar with an unusual anatomy of having a single mesial canal and two distal canals which is in reverse to usually seen, encountered during routine endodontic treatment. The clinician should always keep in mind that anatomic aberrations can occur in any tooth and the recognition of these is achieved by thorough examination of the internal anatomy of the tooth and its radiographs.
\end{abstract}

Keywords: Reverse anatomy, Single mesial canal, Mandibular second molar.

How to cite this article: Giriyappa RH, Majety KK, Gandhi B. Root Canal Treatment of a Mandibular Second Molar with a Reverse Anatomy. World J Dent 2012;3(1):97-99.

\section{Source of support: Nil}

Conflict of interest: None declared

\section{INTRODUCTION}

As rightly quoted by MT Barrett, 'of all the phases of anatomic study in the human system, one of the most complex is the pulp cavity morphology'. The external and internal morphology of the tooth varies from person to person. For each tooth in the permanent dentition, there is a wide range of variation reported in the literature with respect to the frequency of occurrence of the number and shape of the canals in each root, the number of roots and the incidence of molar root fusion. ${ }^{1}$ A number of factors contribute to the variation found in these studies, such as the ethnic background, age of the patient, gender and also the methods used to assess these teeth. ${ }^{2,3}$ As a cause of treatment failures, lack of working knowledge of pulp anatomy ranks second only to errors in diagnosis and treatment planning. Knowledge of the pulp anatomy must be three-dimensional. The pulp cavity must be visualized both longitudinally (from coronal aspect to apical foramen) and in cross section. In addition to general morphologic features, irregularities and 'hidden' regions of the pulp are present within each canal. To clean and shape the pulp system maximally, intracanal instruments must reach as many of these regions as possible to plane the walls to loosen tissue and tissue remnants. ${ }^{4}$ Lack of attention to this important principle may lead to treatment failure. Therefore the purpose of this article is to report the root canal treatment of a mandibular second molar with an unusual anatomy of having two distal canals and one mesial canal which is in contrary to the usual.

\section{CASE REPORT}

A 24-year-old female had reported to the Department of Conservative Dentistry and Endodontics, at KLE VK Institute of Dental Sciences, Belgaum, with a chief complaint of pain in the lower left back tooth region since a week. Her medical history was noncontributory. On examination of the involved region, there was deep caries associated with the tooth $37 ; 36$ was missing. Patient gave a history of extraction of the tooth one-year back owing to carious involvement. There was mesial migration of the tooth 37 .

On examination of the intraoral periapical radiograph (IOPA) of the involved region (Fig. 1), there was radiolucency in the crown region of the tooth 37 involving the pulp. The distal root of the tooth 37 showed two radiolucent lines extending from the pulp chamber to the apex. The root canal morphology confirmed the presence of two canals in the distal root.

An inferior alveolar nerve block was given to the patient on the involved side and under rubber dam isolation an access cavity was prepared of the tooth 37 (Fig. 2).

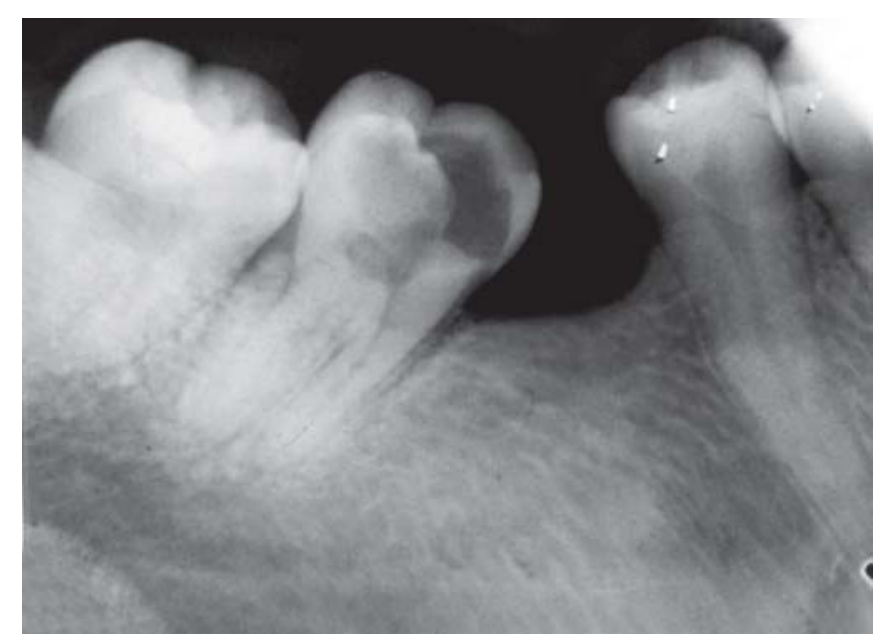

Fig. 1: Preoperative intraoral periapical radiograph 


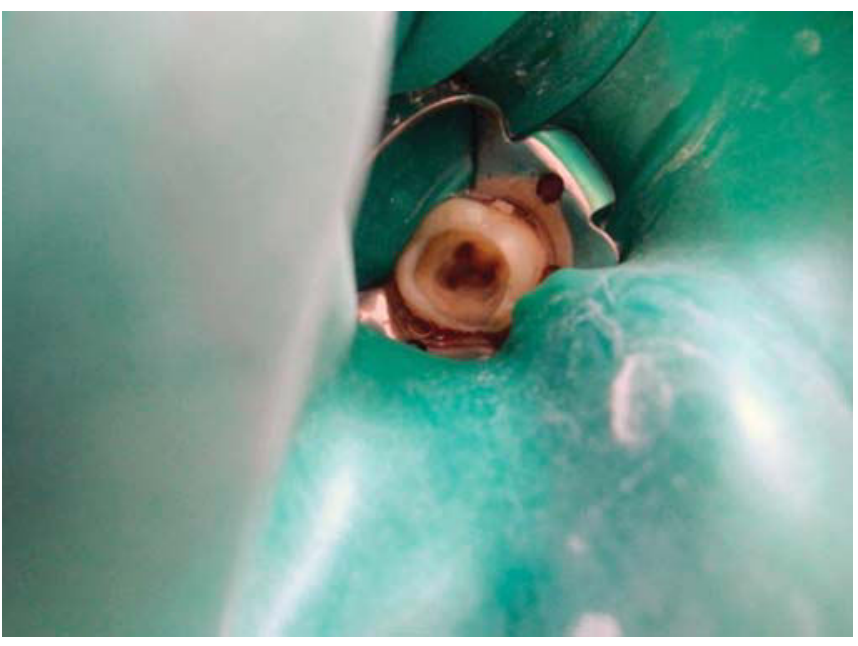

Fig. 2: After access cavity preparation

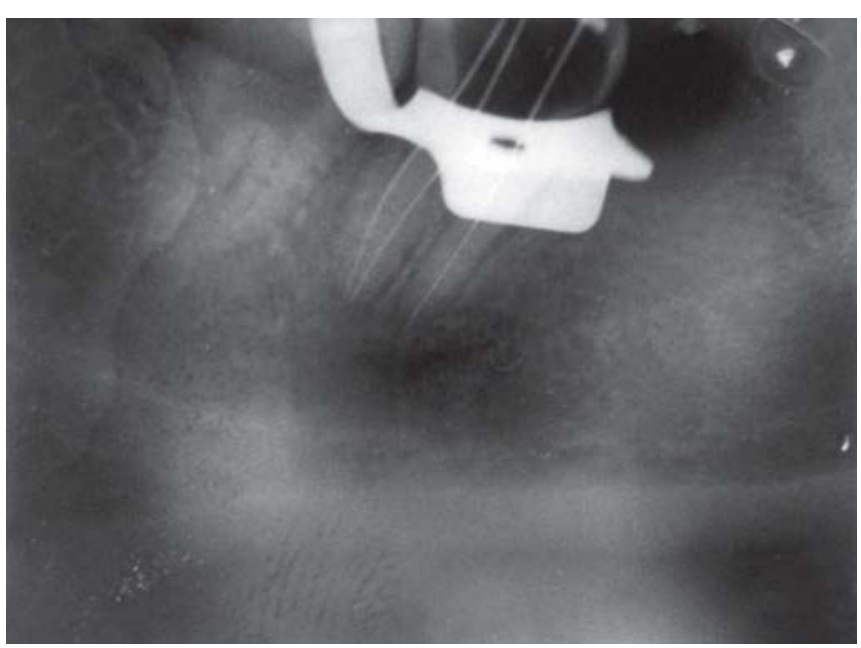

Fig. 3: Working length measurement

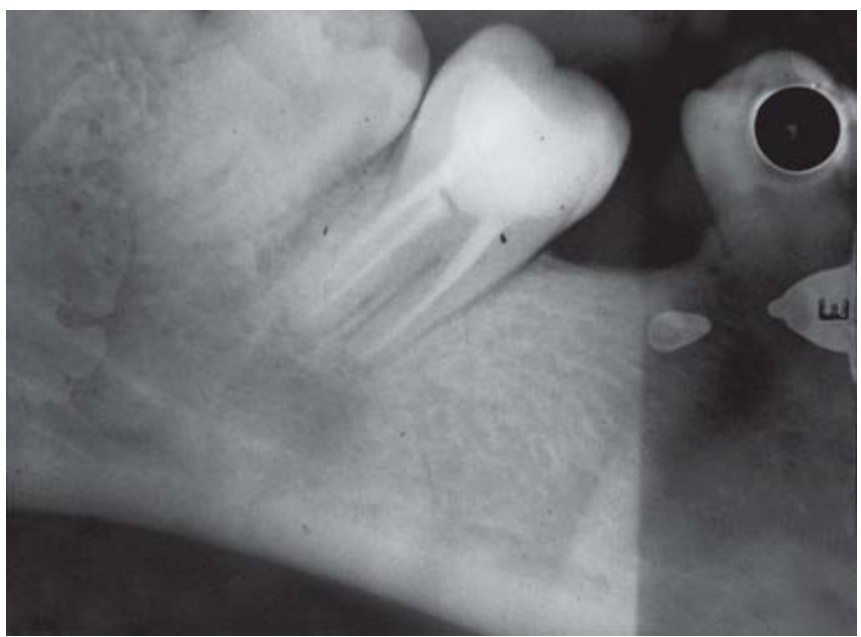

Fig. 4: Postobturation radiograph

The access cavity preparation revealed two canal orifices with the distal root and one canal orifice in the mesial root (Fig. 2).

The pulp was extirpated from all the three canals and working length was determined with ISO $15 \mathrm{~K}$ file using an apex locator and it was confirmed using an IOPA (Fig. 3). The cleaning and shaping was done using the step-back technique and using sodium hypochlorite and normal saline for copious irrigation.

The three canals were enlarged up to a master apical size of ISO $30 \mathrm{~K}$ file. An apical tug back was achieved with an ISO 30 gutta-percha cone and an IOPA was taken for confirming the master cone length.

The canals were then obturated with $\mathrm{AH}$ plus ${ }^{\circledR}$ root canal sealer and gutta-percha using a lateral condensation technique. After the completion of obturation a postendodontic restoration was placed with the tooth 37 using a dual cure hard fluoride releasing core build up material by Pulpdent ${ }^{\circledR}$. A postobturation IOPA was taken (Fig. 4).

\section{DISCUSSION}

The only consistent aspect of the root canal system is its inconsistency. The root canals can take up any configuration and variability is not unusual. The present case described a mandibular second molar with a single mesial canal and two distal canals which is in reverse to the usual anatomy. This case report was similar to another reported by Donald W Wells and William E Bernier. ${ }^{6}$

The usual number of roots and the root canals in the mandibular second molar show two roots $71 \%,{ }^{5} 76 \%{ }^{1}$ and two mesial canals and a single distal canal. The prevalence of two mesial canals is $40 \%,{ }^{4} 89 \%{ }^{1}$ and one distal canal is $92^{4}$ and $95 \%{ }^{1}$

The mesial root of the mandibular second molar has a higher incidence of one canal (14\%) than does the mesial root of the mandibular first molar (4.2\%). ${ }^{1}$ The incidence of one mesial canal at the apex is $60.3^{1}$ and $25 \%{ }^{4}$ The incidence of two distal canals in the mandibular second molar at mid-root level is $14.9^{1}$ and $5 \%{ }^{4}$ The incidence of two distal canals at apex is $5^{1}$ and $3 \%{ }^{4}$

It is important to visualize and to have knowledge of internal anatomy relationships before undertaking endodontic therapy. Gaining knowledge of anatomy from textbooks is the most important and most useful method of learning. Common and frequent variations must be memorized for each tooth. It is useful to know the approximate percentage of the number of roots, number of canals per root and their location and also their frequent curvatures. ${ }^{9}$

Careful evaluation of two or more periapical radiographs is mandatory. These angled radiographs provide much needed information about root canal morphology. ${ }^{7}$ Friedman et al stressed the importance of radiographs 'that any attempt to develop techniques that require fewer radiographs runs the risk of missing information which may be significant for the success of therapy' ${ }^{8}$ 
Diagnostic measures, such as multiple preoperative radiographs, examination of the pulp chamber floor with a sharp explorer, troughing of grooves with ultrasonic tips, staining the chamber floor with $1 \%$ methylene blue dye, performing the sodium hypochlorite 'champagne bubble' test and visualizing canal bleeding points are important aids in locating root canal orifices. ${ }^{8}$

An important aid for locating root canals is the dental operating microscope (DOM) which was introduced into endodontics to provide enhanced lighting and visibility. ${ }^{8,10}$ It brings minute details into clear view.

\section{SUMMARY}

The only source of knowledge is experience and it alone teaches the variability among the normal. There are numerous cases in the literature regarding the root canal anatomy of the mandibular second molar. The occurrence of a single mesial canal is 60.3 and $25 \%$ as per various studies and that of two distal canals is 5 and $3 \%$. The key success to endodontic treatment is through debridement of the entire root canal system and recognizing the aberration leads to long-term clinical success.

\section{REFERENCES}

1. Ingle, Bakland, Baumgartner. Morphology of teeth and their root canal systems Endodontics (6th ed), Chapter 6 151-220.

2. Fava LR. Root canal treatment in an unusual maxillary first molar: A case report. Int Endod J 2001;34:649-53.
3. Cleghorn B, Christie W, Dong C. Root and root canal morphology of the human permanent maxillary first molar: A literature review. J Endod 2006;32:813-21.

4. Mahmoud Torabinejad, Richard E Walton. Endodontics principles and practice. (4th ed). Chapter 13, Internal Anatomy 217-239; Appendix A: Pulpal anatomy and access preparation.

5. Grossman's Endodontic Practice, 12th edition, Chapter 9: Anatomy of pulp cavity and its access opening, 176-220.

6. Donald W Wells, William E Bernier. A single mesial canal and two distal canals in mandibular second molar. Journal of Endodontics 1984;10(8):400-03.

7. Frank J Vertucci. Root canal morphology and its relationship to endodontic procedures. Endodontic Topics 2005;10:3-29.

8. Friedman S, Moshonov J, Stabholz A. Five root canals in a mandibular first molar. Dent Traumatol 1986;2:226-28.

9. Vertucci F. Root canal anatomy of the human permanent teeth. Oral surg Oral Med Oral Pathol 1984;58:589.

10. Dean Baugh, James Wallace. Middle mesial canal of the mandibular first molar: A case report and literature review. Journal of Endodontics March 2004;30(3).

\section{ABOUT THE AUTHORS}

\section{Ramesh Halebathi Giriyappa (Corresponding Author)}

Reader, Department of Conservative Dentistry and Endodontics, KLE VK Institute of Dental Sciences, Belgaum, Karnataka, India e-mail: halebathi@yahoo.com

\section{Kishore Kumar Majety}

Senior Lecturer, Department of Conservative Dentistry and Endodontics, KLE VK Institute of Dental Sciences, Belgaum Karnataka, India

\section{Bhavana Gandhi}

Postgraduate Student, Department of Conservative Dentistry and Endodontics, KLE VK Institute of Dental Sciences, Belgaum Karnataka, India 